Fetal Diagnosis and Therapy

\title{
Prenatal Identification of Pierre Robin Sequence: A Review of the Literature and Look towards the Future
}

\author{
Matthew G. Kaufman ${ }^{a}$ Christopher l. Cassady ${ }^{b}$ Charles H. Hyman ${ }^{a}$ \\ Wesley Lee ${ }^{c}$ Mehernoor F. Watchad Helena K. Hippard ${ }^{d}$ Olutoyin A. Olutoye ${ }^{d}$ \\ David Y. Khechoyan ${ }^{a}$ Laura A. Monson ${ }^{\text {a }}$ Edward P. Buchanan ${ }^{a}$ \\ ${ }^{a}$ Division of Plastic Surgery, Michael E. DeBakey Department of Surgery, and Departments of b Radiology, \\ cObstetrics and Gynecology and d Pediatric Anesthesiology, Baylor College of Medicine, Houston, Tex., USA
}

\section{Key Words}

Pierre Robin sequence · Prenatal identification

\begin{abstract}
Fetal ultrasonography is an important tool used to prenatally diagnose many craniofacial conditions. Pierre Robin sequence (PRS) is a rare congenital deformation characterized by micrognathia, glossoptosis, and airway obstruction. PRS can present as a perinatal emergency when the retropositioned tongue obstructs the airway leading to respiratory compromise. More predictable and reliable diagnostic studies could help the treating medical team as well as families prepare for these early airway emergencies. The medical literature was reviewed for different techniques used to prenatally diagnose PRS radiologically. We have reviewed these techniques and suggested a possible diagnostic pathway to consistently identify patients with PRS prenatally.
\end{abstract}

(c) 2015 S. Karger AG, Basel

\section{KARGER}

(C) 2015 S. Karger AG, Basel

$1015-3837 / 15 / 0392-0081 \$ 39.50 / 0$

E-Mail karger@karger.com

www.karger.com/fdt

\section{Introduction}

Pierre Robin sequence (PRS) is classically defined as a congenital deformation consisting of micrognathia, glossoptosis, and airway obstruction [1]. Cleft palate is also found in roughly $90 \%$ of patients with PRS [2]. While the sequence is relatively rare with a reported prevalence ranging from $1: 2,000$ to $1: 30,000$ of live births, the mortality rate in the condition has been reported to be as high as 30\% [3-6]. The most concerning findings in PRS patients are micrognathia and the retropositioned tongue, as the combination of these two can obstruct the airway causing respiratory distress and poor oral feeding. Prenatal suspicion of PRS is essential for preparing the delivery team for a possible airway emergency. Because PRS is based on clinical findings, it cannot be definitively diagnosed until delivery and demonstration of airway compromise.

Ultrasonography is currently the most described modality to screen for PRS. Ultrasound examination of the fetus is the current standard for prenatal care and the pri-

Edward P. Buchanan, MD

Division of Plastic Surgery, Michael E. DeBakey Department of Surgery Baylor College of Medicine, 6701 Fannin St, CC 610.00

Houston, TX 77030 (USA)

E-Mail ebuchana@bcm.edu 
mary imaging modality for both screening and diagnostic purposes. It allows for identification of structural abnormalities that may serve as harbingers for other phenotypic anomalies, syndromes, or genetic abnormalities. Examination of the fetal face is performed using a twodimensional ultrasound probe in multiple planes. A coronal view is used to see soft tissues, bones, and overall symmetry of the face. A sagittal view provides the examiner with a view of the fetal forehead, nasal bridge, and mandible. The transverse view provides visualization of the mandible, maxilla, and palate [7].

The medical literature describing prenatal findings in PRS has been limited. Attempts at prenatal diagnosis have centered on retrospective reviews examining features of the sequence, including micrognathia, retrognathia, glossoptosis, and polyhydramnios, which have largely been based on prenatal ultrasound findings. This paper will review and discuss the literature pertaining to the prenatal radiologic findings in PRS. Furthermore, a potential pathway for a future screening tool will be discussed.

\section{Methods and Data Sources}

A systematic review of the literature concerning PRS and prenatal diagnosis was performed. PubMed and SCOPUS databases were searched for articles published dealing with prenatal radiologic diagnosis of PRS. The following search terms were used: (Pierre OR Robin) AND (micrognathia OR retrognathia OR glossoptosis) AND (ultrasound OR MRI OR imaging OR radiology) AND (prenatal OR antenatal OR fetus OR fetal). PubMed returned 20 search results, and SCOPUS returned 26 search results, which were screened for relevance. Only papers written in English were included. Prenatal findings in the context of PRS were included. After evaluating the search results for relevance, 11 articles met the inclusion criteria and were included in this review. References from the resultant publications were examined for relevance within the constraints of the above criteria.

\section{Micrognathia}

Most of the current medical literature has attempted to prenatally diagnose PRS primarily by identifying micrognathia as an isolated finding. Micrognathia, which describes an undersized mandible, is often confused with retrognathia, a term that describes a retropositioned mandible [1]. In PRS, the micrognathia is so severe that it results in retrognathia, both in the vertical and horizontal facial planes. This leads to glossoptosis, a posteriorly rotated tongue blocking the airway.
Micrognathia is typically diagnosed without using definitive metrics. Subjective diagnosis has been documented as early as 13 weeks during routine fetal anatomical survey [8]. Using these methods, a retrospective analysis of 20 cases of PRS was performed. Only 11 (55\%) of these patients were diagnosed with micrognathia. The authors attributed the low sensitivity to diagnostic error [9]. In a prospective study of 210 fetuses using qualitative diagnostic criteria for micrognathia, and without consideration for PRS, there was a sensitivity of $72.7 \%$ and a specificity of $99.2 \%$ [10].

Numerous studies using more standardized objective methods for measuring fetal jaw size and micrognathia have been performed [11-20]. Paladini et al. [14] introduced the concept of a jaw index by defining growth curves and biometry in order to establish normal fetal jaw size and dimensions in utero. Two vectors of growth were calculated by measuring two diameters of the mandible at two different time periods, at 12 and again at 37 weeks. The jaw index was determined by dividing the anteroposterior mandibular distance by the biparietal skull distance. The authors performed a prospective study, using a jaw index cutoff of 23 (2 standard deviations below the mean) to determine micrognathia. This metric demonstrated a sensitivity of $100.0 \%$ and a specificity of $98.1 \%$. While accurate, it has been noted that the jaw index is both more difficult and time consuming to measure accurately than more recently developed measures [21].

Rotten et al. [15] diagnosed micrognathia by comparing the widths of the mandible to the maxilla. The widths were measured from three-dimensional reconstructions. Mandibular (MD) and maxillary widths (MX) were measured on a line $10 \mathrm{~mm}$ posterior to the anterior osseous border on axial view. MD and MX were measured for 245 normal fetuses between 18 and 28 weeks' gestational age. The MD/MX ratio was then calculated, and micrognathia was defined as $<2$ standard deviations from the mean $(<0.785)$. The MD/MX ratio was then calculated from the three-dimensional reconstructions of 8 fetuses that were diagnosed with PRS postnatally. Only $50 \%(n=4)$ of these cases had MD/MX ratios consistent with micrognathia $(<0.785)$.

The inferior facial angle (IFA) was introduced to help better define retrognathia in utero (fig. 1) [22]. The IFA is calculated by measuring the angle made by the crosssection of a line orthogonal to the forehead at the level of the nasofrontal suture and a line from the tip of the mentum to the anterior border of the more protrusive lip on a sagittal view.
Kaufman et al. 
In studying 371 normal fetuses, the mean IFA value was determined to be $65.5 \pm 8.13^{\circ}$ over the $18-28$ weeks' gestational age interval. The study defined retrognathism as an IFA value $<49.2^{\circ}$ In all 8 patients diagnosed with PRS in the study, the IFA was 2 standard deviations below the mean $\left(35-46^{\circ}\right)$, suggesting that retrognathia might be a more accurate predictor for prenatal suspicion of PRS (fig. 2).

Palit et al. [19] introduced the fronto-naso-mental (FNM) angle as another method of measuring retrognathia that shows consistency with the postnatal diagnosis of PRS. The FNM angle is determined by measuring the angle made by the intersection of a line from the most prominent point of the forehead to the tip of the nose and a line from the mentum to the tip of the nose in a sagittal view (fig. 3). The mean FNM angle of 81 fetuses between 18 and 22 weeks' gestational age was $146.4^{\circ}$. Retrognathia was defined as any measurement falling below the 5 th percentile of $142^{\circ}$. While this metric does not measure the size of the mandible and does not specifically evaluate for micrognathia, it was accurate in correctly predicting PRS in 4 fetuses who all fell below the 5th percentile, with an average angle of $119.25^{\circ}$. The authors used two-dimensional views for measurements, but noted that three-dimensional multiplanar imaging reduces the likelihood of measuring the incorrect angle.

Luedders et al. [21] retrospectively analyzed 58 cases of postnatally diagnosed micrognathia using IFA and FNM angles. Fifty-four of the 58 cases $(93 \%)$ were validated using the cutoffs of $142^{\circ}$ for the FNM angle and $50^{\circ}$ for the IFA. The mean FNM angle and IFA were 123.3 and $44.8^{\circ}$, respectively. While relatively specific, the combination of the two was not as sensitive, mainly because the FNM angle was below the cutoff in many of the control cases. This series evaluated only 5 cases of PRS, all of which were confirmed using both metrics [21].

Sepulveda et al. [23] used the absence of a mandibular gap in the retronasal triangle view of the mandible as a marker for micrognathia in first-trimester fetuses. This is the first objective measure to consistently diagnose micrognathia early in pregnancy. The retronasal triangle view is seen in the coronal plane of the face where the primary palate and frontal processes of the maxilla are both visible. Normal first-trimester fetuses have a 'mandibular gap' between the left and right bodies of the mandible. This gap can be seen with both two- and three-dimensional ultrasonography. The mandibular gap was prospectively and retrospectively measured in a total of 204 normal fetuses at 11-13 weeks and compared to $12 \mathrm{fe}$ tuses with suspected micrognathia. All normal fetuses demonstrated a mandibular gap. Among the 12 fetuses

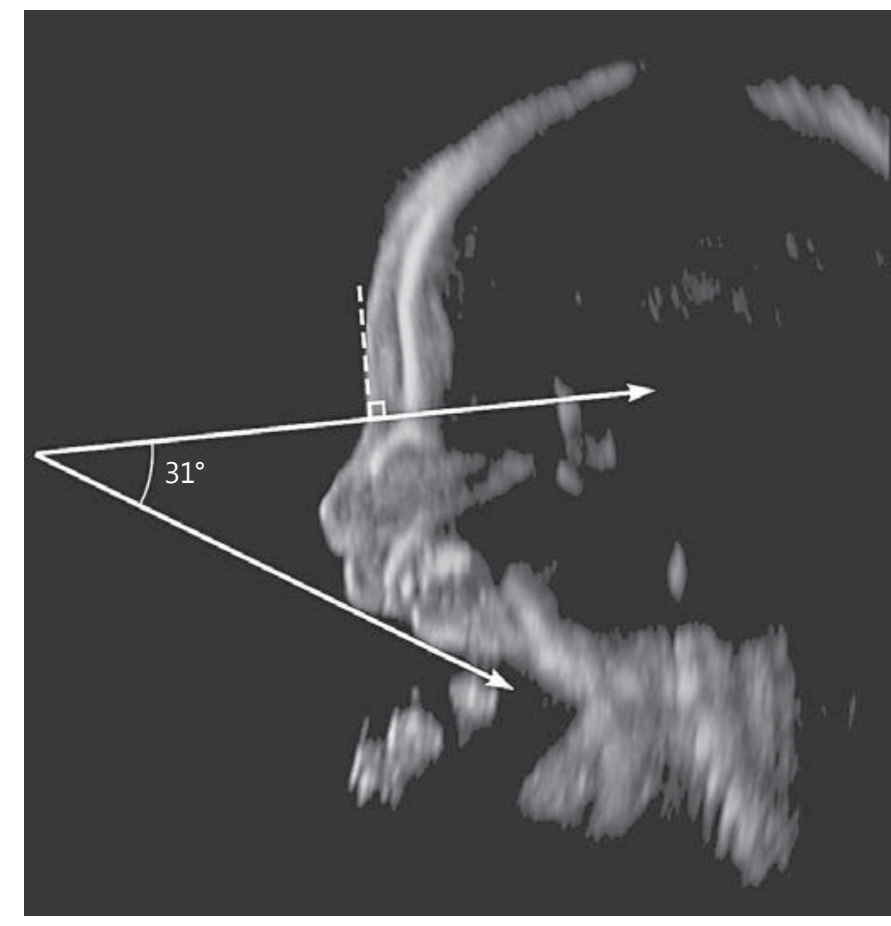

Fig. 1. Two-dimensional ultrasound of a fetal facial profile with micrognathia with the IFA demonstrated.

with suspected micrognathia, 9 could be accurately measured, and all had an absent mandibular gap. Though scanning of the jaw is generally not routinely performed in the first trimester, this technique offers the ability to detect micrognathia earlier than any other technique. Whether this helps to predict PRS is unknown.

Recently, Nemec et al. [24] performed a retrospective study that aimed to diagnose micrognathia with magnetic resonance imaging (MRI) using previously established ultrasound diagnostic criteria. This study compared the MRI findings of 355 fetuses without facial abnormalities seen on ultrasound to 10 fetuses with postnatal or autopsy-confirmed mandibular abnormalities. Of the 10 fetuses with abnormalities, 2 were diagnosed with PRS. From the MRI images, the study obtained both objective measurements (IFA, anteroposterior mandibular distance, biparietal skull distance, and jaw index) as well as subjective assessment of a receding chin from the MRI images. The IFA was determined in 334 of the 355 (94.1\%) normal fetuses studied, and the data was confirmed using an IFA of $50^{\circ}$ for the lower limit of normal as all 10 fetuses with mandibular abnormalities had IFA values $<50^{\circ}$. The jaw index was measured in 292 of the 355 (82.3\%) normal fetuses studied, and a significant difference was found between 
Fig. 2. Two-dimensional ultrasound images of a normal fetal facial profile (a) and a patient with micrognathia (b). The lines to measure the IFA have been drawn to assist with rapid evaluation of micrognathia.
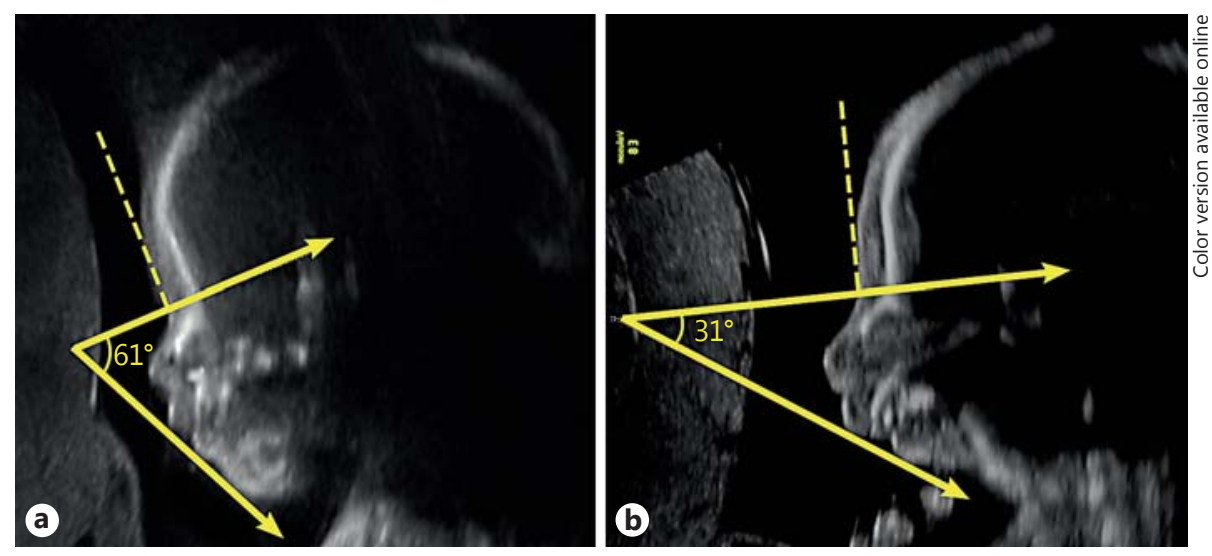

Fig. 3. Two-dimensional ultrasound images of a normal fetal facial profile (a) and a patient with micrognathia (b). The lines to measure the FNM angle have been drawn.
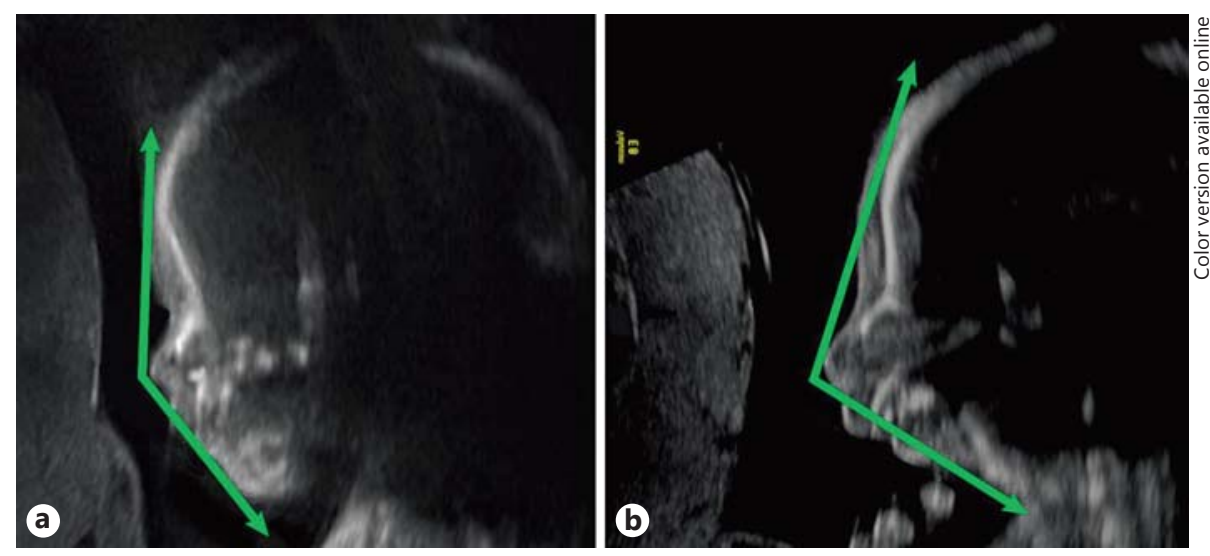

MRI and ultrasound measurements. Still, a jaw index was used to distinguish between micro- and retrognathia, with micrognathic fetuses defined as having IFA values $<50^{\circ}$ and jaw index values below the 5th percentile. The study was also able to demonstrate high intrareader and interreader agreement for each of the measurements, as well as in the subjective determination of severely recessed mandibles.

\section{Glossoptosis}

While many methods have been introduced to prenatally diagnose micro- and retroganthia, their utility in predicting PRS has not been validated. Whereas micrognathia is the defining anatomic feature in PRS and has received much attention, glossoptosis, the second and critical clinical finding, has received very little.

Bronshtein et al. [25] examined the ability to predict PRS by measuring glossoptosis via ultrasonography. They evaluated for glossoptosis by viewing the fetus in profile to watch the echogenic tongue for $20-30 \mathrm{~min}$ to see if it was either posteriorly displaced or reached the anterior mandibular alveolar ridge during normal movements. If glossoptosis was suspected, micrognathia was subjectively measured to confirm PRS. They were retrospectively able to visualize glossoptosis and subjective micrognathia on 4 confirmed cases in infants diagnosed with PRS after delivery. They prospectively tested 8,000 consecutive pregnancies and found 2 cases of glossoptosis and micrognathia, one of which was postnatally confirmed to have PRS (the second case was not able to undergo a reliable postmortem examination due to the destructive nature of the termination procedure). The Bronshtein study did not have any false positives, and the tongue in all of the normal cases reached the anterior mandibular alveolar ridge. This measurement shows promise as a way to differentiate PRS from isolated micrognathia.

\section{Polyhydramnios}

The first suspected prenatal case of PRS was reported by Pilu et al. [26], in which micrognathia and polyhydramnios were described together. It is postulated that 
polyhydramnios occurs as a result of swallowing difficulty secondary to the obstruction caused by micrognathia and glossoptosis. The literature is limited with respect to this potential association, but there are case reports that describe patients with both polyhydramnios and micrognathia, indicating that further work is needed to establish a pathogenetic mechanism [27-29]. In a retrospective analysis of 20 infants diagnosed with PRS by Hsieh et al. [9], only 12 demonstrated polyhydramnios on ultrasound. Bromley and Benacerraf [30] found that polyhydramnios was not unique to PRS; it occurred in 13 of the 20 fetuses sonographically diagnosed with micrognathia unrelated to PRS.

Interestingly, a case report by Aggarwal and Kumar [31] suggested that oligohydramnios may be a causative factor in the development of PRS, as decrease in amniotic fluid may lead to mechanical deformational forces on the developing facial architecture. As there is no clear consensus on the role amniotic fluid has on PRS, there seems to be no benefit to incorporate these findings into a screening algorithm.

\section{Discussion}

PRS is defined as micrognathia, glossoptosis, and airway obstruction. Because one of these findings can only be determined after delivery, prenatal diagnosis remains a challenge. Micrognathia, retrognathia, and glossoptosis have been found to be useful predictors of PRS, but no definitive parameters or combination of findings have been described that consistently and accurately assist with the diagnosis. Research efforts evaluating the prenatal radiologic findings specific to PRS are diverse and consist largely of retrospective case studies and reports.

Measuring and defining micro- and retrognathia has been extensively studied to help predict PRS. The two most promising metrics are the IFA and FNM angles. These two parameters have been studied independently and in comparison with one another. While they have been shown to be specific in diagnosing retrognathia, they have not been shown to be sensitive, according to the parameters established by Luedders et al. [21]. However, the FNM angle has been associated with more false positives, and as mentioned above, the combination of IFA and FNM angle was not as sensitive as the use of either alone. Furthermore, there has been very weak evidence linking these measurements to the diagnosis of PRS, and neither is used as a consistent and reliable diagnostic tool. Because of its higher sensitivity and specificity, the IFA

Prenatal Identification of Pierre Robin Sequence could potentially serve as an initial screening tool for diagnosing mandibular abnormalities.

Compared to retrognathia and micrognathia, glossoptosis has been studied sparingly. Bronshtein et al. [25] evaluated subjectively for glossoptosis as well as micrognathia using ultrasound, and their findings indicate a potentially strong predictor of PRS. Their method of diagnosing glossoptosis requires a considerable amount of time and may be impractical in the clinical setting. A simpler and more efficient method for identifying glossoptosis may be extremely helpful for future diagnostic efforts.

Many approaches have been described in an attempt to prenatally diagnose PRS. Previous, mostly retrospective, studies have been too small in sample size, lacking specificity or sensitivity, to demonstrate any clear diagnostic algorithm. If identified, an accurate and reproducible screening examination would allow for prenatal diagnosis that could prepare the treating team to deal with the postnatal care challenges and the family with prenatal education. Only one study has aimed to establish a prospective screening algorithm, but there has been no follow-up data regarding its efficacy [25]. Furthermore, the methods used increased the time required to perform prenatal ultrasound by at least 20-30 min. An accurate, consistent, and expeditious screening tool that can be easily incorporated into the current prenatal ultrasound evaluation would be ideal in helping with diagnosis. Such a screening tool would need to be validated prospectively to help eliminate any biases inherent in retrospective studies. The majority of the cases reported in the literature were retrospectively analyzed after postnatal diagnosis. The challenges facing a prospective trial relate to the relative rarity of the condition. Out of 28,935 ultrasounds, Luedders et al. [21] identified only 58 fetuses with micrognathia and 4 with PRS.

Recent work has been done to demonstrate the prenatal utility of MRI for the diagnosis of craniofacial abnormalities, including cleft lip and palate [32-34]. While MRI could potentially offer many benefits with respect to prenatal morphologic findings (i.e. reproducible, highresolution three-dimensional images of the face, palate, and jaw with no additional radiation exposure), its role in a screening algorithm has not been investigated. Limitations include that both the mandible and its adjacent musculature have similar MRI signal intensities on T2weighted imaging, making the mandible less easy to distinguish than on ultrasound.

Sagittal MRI sequences of the fetal face are actually easily obtainable, and the position of the fetal tongue is straightforwardly assessed because of the high contrast 
Fig. 4. Fetal MRI images of a normal facial profile (a) and a patient with micrognathia and glossoptosis (b). The arrow in a demonstrates the appearance of the tongue positioned on the floor of the mouth, not obstructing the airway. The arrow in $\mathbf{b}$ demonstrates a retropositioned tongue, with the base of the tongue positioned in the fetal airway.
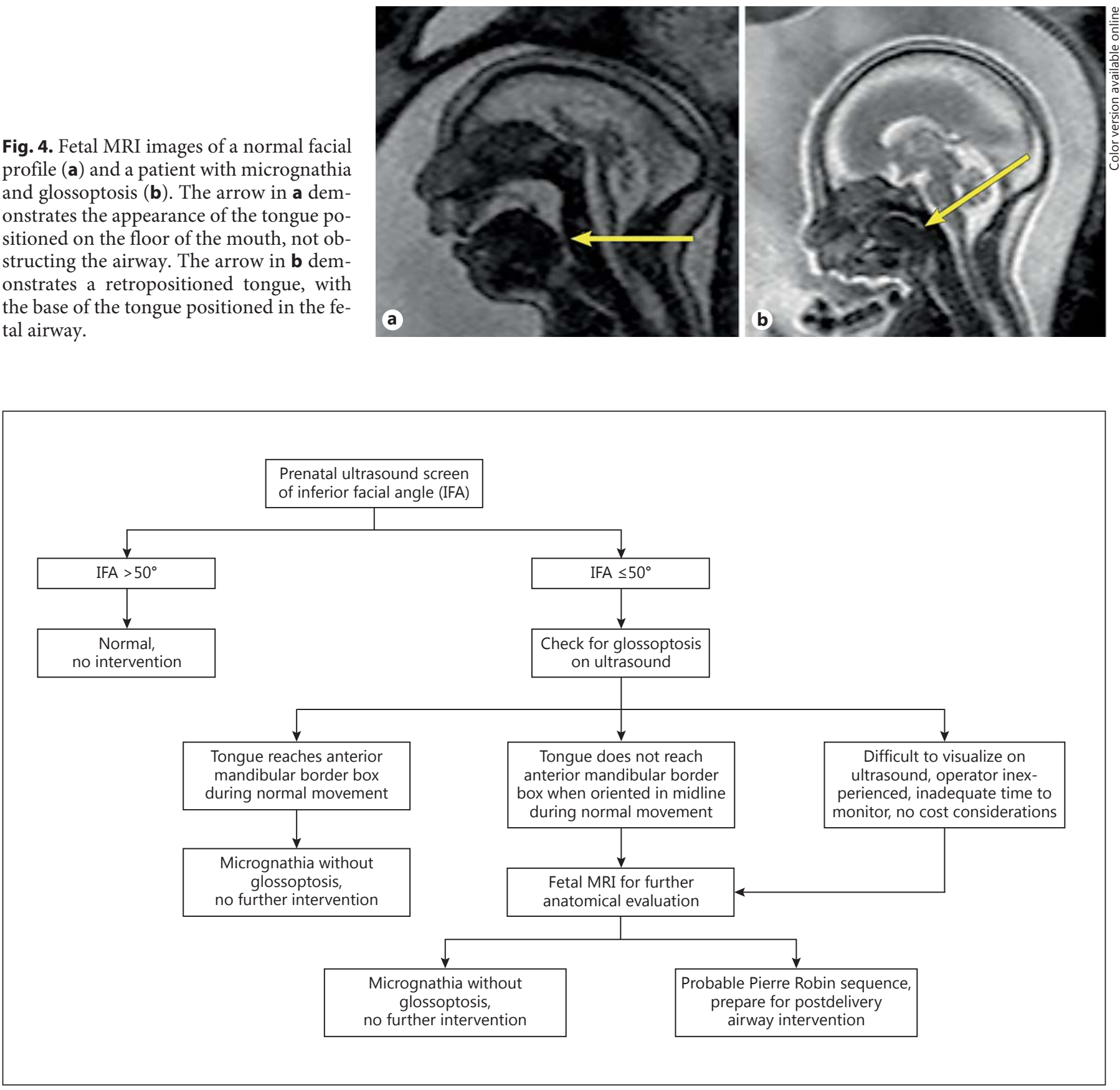

Fig. 5. Proposed screening algorithm for the prenatal identification of PRS.

conspicuity difference between the low-signal muscle of the tongue and the high-signal amniotic fluid in the mouth and oropharynx on T2-weighted images. Similarly, MRI is able to outline posterior palate defects against the high-signal fluid in the nasopharynx, indicating that MRI assessment of glossoptosis may have advantages over ultrasound (fig. 4). Due to issues of additional cost and resource allocation, it would seem impractical to use MRI as a first-line screening modality, though it could become a useful adjuvant to diagnosing facial structure pathology in cases with suggestive ultrasound findings. Whether or not one would choose to evaluate for glos- 


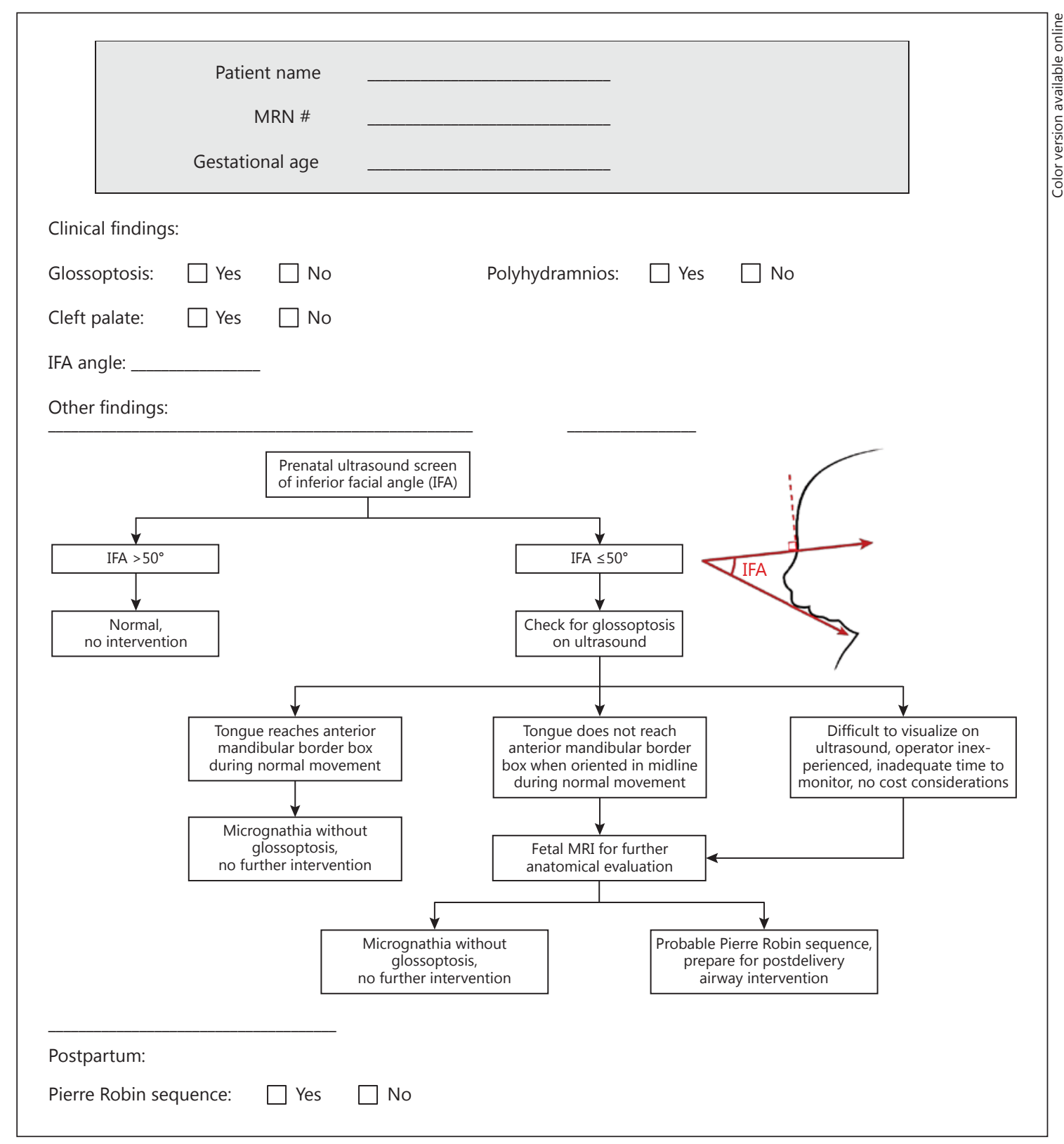

Fig. 6. Screening worksheet for the prenatal identification of PRS.

soptosis in suspicious cases by ultrasound or MRI depends on a variable number of considerations, including the experience or confidence of the ultrasonographer, time constraints, fetal position, resource availability, and cost.

An important aspect of developing a prenatal screening algorithm is to incorporate the previously mentioned measurement techniques into the standard prenatal ultrasound schedule that all gravid women undergo. This would require no extra maternal clinic visits or even require any additional ultrasound examinations. Evaluating for cleft palate, which can be associated with up to $90 \%$ of PRS cases, is currently standard of care in prenatal ultrasound screening even if findings more specific to PRS are not. We believe an accurate diagnostic approach for PRS would most likely involve initial screening for retrognathia/micrognathia by measuring the fetal IFA along with the routine imaging of these checkup visits, as 
sagittal or 'profile' views are a typical component of anatomy scans. Because of the high specificity and sensitivity for micrognathia, an abnormal IFA could then prompt the ultrasonographer to look for glossoptosis, a more specific predictive indicator of PRS. In cases where the findings are ambiguous, adjunct imaging with MRI may prove beneficial. Although additional ultrasound findings (polyhydramnios and/or cleft palate) have been associated with PRS, their low specificity and rather ubiquitous presence in the absence of PRS makes them less specific diagnostic indicators of the sequence. With these issues in mind, we propose the algorithm shown in figure 5 , along with a sample worksheet designed to be used by ultrasonographers (fig. 6).

For validation of our screening algorithm, a prospective study with sufficient statistical power would need to be performed at a high-volume institution over many years, or collectively in a multi-institutional trial, and would only require minimal change to the current prenatal imaging evaluation. A multidisciplinary effort, includ- ing specialists from the fields of maternal-fetal medicine, radiology, anesthesiology, and plastic surgery, would be required to make such an endeavor successful.

\section{Conclusion}

Prenatal diagnosis of PRS would be an advance in current clinical practice by allowing for appropriate prenatal consultation as well as intra- and postpartum care. Currently, no screening algorithm exists for a consistent and reproducible diagnosis. Although the medical literature has identified several important measurements to assist with diagnosis, these need to be tailored into a clinically applicable screening tool and validated with prospective clinical trials. By developing a reliable screening protocol, early risk stratification can be achieved with the hopes of decreasing perinatal morbidity. In the setting and spirit of multidisciplinary care and patient-centered outcomes, the goal of prenatal diagnosis of PRS can be achieved.

\section{References}

$\checkmark 1$ Breugem CC, Courtemanche DJ: Robin sequence: clearing nosologic confusion. Cleft Palate Craniofac J 2010;47:197-200.

-2 Evans KN, Sie KC, Hopper RA, Glass RP, Hing AV, Cunningham ML: Robin sequence: from diagnosis to development of an effective management plan. Pediatrics 2011;127:936-948.

-3 Caouette-Laberge L, Bayet B, Larocque Y: The Pierre Robin sequence: review of 125 cases and evolution of treatment modalities. Plast Reconstr Surg 1994;93:934-942.

4 Jones KL: Facial defects as a major feature; in Jones KL (ed): Smith's Recognizable Patterns of Human Malformations, ed 5. Philadelphia, WB Saunders, 1997, pp 234-235.

5 Holder-Espinasse M, Abadie V, CormierDaire V, Beyler C, Manach Y, Munnich A, Lyonnet S, Couly G, Amiel J: Pierre Robin sequence: a series of 117 consecutive cases. J Pediatr 2001;139:588-590.

6 van den Elzen AP, Semmerkrot BA, Bongers EM, Huygen PL, Marres HA: Diagnosis and treatment of the Pierre Robin sequence results of a retrospective clinical study and review of the literature. Eur J Pediatr 2001;160:47-53.

-7 Kurjak A, Azumendi G, Andonotopo W, Salihagic-Kadic A: Three- and four-dimensional ultrasonography for the structural and functional evaluation of the fetal face. Am J Obstet Gynecol 2007;196:16-28.

-8 Teoh M, Meagher S: First】trimester diagnosis of micrognathia as a presentation of Pierre Robin syndrome. Ultrasound Obstet Gynecol 2003;21:616-618.
9 Hsieh YY, Chang CC, Tsai HD, Yang TC, Lee $\mathrm{CC}$, Tsai $\mathrm{CH}$ : The prenatal diagnosis of Pierre-Robin sequence. Prenat Diagn 1999; 19:567-569.

10 Paladini D: Fetal micrognathia: almost always an ominous finding. Ultrasound Obstet Gynecol 2010;35:377-384.

11 Otto C, Platt LD: The fetal mandible measurement: an objective determination of fetal jaw size. Ultrasound Obstet Gynecol 1991;1:12-17.

12 Chitty LS, Campbell S, Altman DG: Measurement of the fetal mandible - feasibility and construction of a centile chart. Prenat Diagn 1993;13:749-756.

13 Watson WJ, Katz VL: Sonographic measurement of the fetal mandible: standards for normal pregnancy. Am J Perinatol 1993;10:226228.

14 Paladini D, Morra T, Teodoro A, Lamberti A, Tremolaterra F, Martinelli P: Objective diagnosis of micrognathia in the fetus: the jaw index. Obstet Gynecol 1999;93:382-386.

15 Rotten D, Levaillant JM, Martinez H, Ducou le Pointe H, Vicaut E: The fetal mandible: a $2 \mathrm{D}$ and $3 \mathrm{D}$ sonographic approach to the diagnosis of retrognathia and micrognathia. Ultrasound Obstet Gynecol 2002;19:122-130.

16 Zalel Y, Gindes L, Achiron R: The fetal mandible: an in utero sonographic evaluation between 11 and 31 weeks' gestation. Prenat Diagn 2006;26:163-167.

17 Borenstein M, Persico N, Strobl I, Sonek J, Nicolaides KH: Frontomaxillary and mandibulomaxillary facial angles at $11+0$ to $13+6$ weeks in fetuses with trisomy 18. Ultrasound Obstet Gynecol 2007;30:928-933.

18 Roelfsema NM, Grijseels EW, Hop WC Wladimiroff JW: Three-dimensional sonography of prenatal skull base development. Ultrasound Obstet Gynecol 2007;29:372-377.

19 Palit G, Jacquemyn Y, Kerremans M: An objective measurement to diagnose micrognathia on prenatal ultrasound. Clin Exp Obstet Gynecol 2008;35:121-123.

20 de Jong-Pleij EAP, Ribbert LSM, Manten GTR, Tromp E, Bilardo CM: Maxilla-nasionmandible angle: a new method to assess profile anomalies in pregnancy. Ultrasound Obstet Gynecol 2011;37:562-569.

21 Luedders DW, Bohlmann MK, Germer U, Axt-Fliedner R, Gembruch U, Weichert J: Fetal micrognathia: objective assessment and associated anomalies on prenatal sonogram. Prenat Diagn 2011;31:146-151.

22 Merrifield LL: The profile line as an aid in critically evaluating facial esthetics. Am J Orthod 1966;52:804-822.

23 Sepulveda W, Wong AE, Als FV, Andreeva E, Adzehova N, Martinez-Ten P: Absent mandibular gap in the retronasal triangle view: a clue to the diagnosis of micrognathia in the first trimester. Ultrasound Obstet Gynecol 2012;39:152-156.

-24 Nemec U, Nemec SF, Brugger PC, Weber M, Bartsch B, Bettelheim D, Gruber M, Prayer D: Normal mandibular growth and diagnosis of micrognathia at prenatal MRI. Prenat Diagn 2015;35:108-116. 
25 Bronshtein M, Blazer S, Zalel Y, Zimmer EZ: Ultrasonographic diagnosis of glossoptosis in fetuses with Pierre Robin sequence in early and mid pregnancy. Am J Obstet Gynecol 2005;193:1561-1564.

26 Pilu G, Reece EA, Romero R, Bovicelli L, Hobbins JC: Prenatal diagnosis of craniofacial malformations with ultrasonography. Am J Obstet Gynecol 1986;155:45-50.

27 Matsumoto M, Yanagihara T, Hanaoka U, Maesato T, Kuno A, Yamashiro C, Tanaka H, Hayashi K, Hata T: Antenatal three-dimensional sonographic features of Pierre-Robin syndrome. Case report. Gynecol Obstet Invest 2001;51:141-142.
28 Morokuma S, Anami A, Tsukimori K, Fukushima $\mathrm{K}$, Wake N: Abnormal fetal movements, micrognathia and pulmonary hypoplasia: a case report. BMC Pregnancy Childbirth 2010;10:46.

29 Soulier M, Sigaudy S, Chau C, Philip N: Prenatal diagnosis of Pierre-Robin sequence as part of Stickler syndrome. Prenat Diagn 2002; 22:567-568.

30 Bromley B, Benacerraf BR: Fetal micrognathia: associated anomalies and outcome. J Ultrasound Med 1994;13:529-533.

31 Aggarwal S, Kumar A: Fetal hydrocolpos leading to Pierre Robin sequence: an unreported effect of oligohydramnios sequence. J Perinatol 2003;23:76-78.
32 Hosny IA, Elghawabi HS: Ultrafast MRI of the fetus: an increasingly important tool in prenatal diagnosis of congenital anomalies. Magn Reson Imaging 2010;28:1431-1439.

33 Manganaro L, Tomei A, Fierro F, Di Maurizio M, Sollazzo P, Sergi ME, Vinci V, Bernardo S, Irimia $\mathrm{D}$, Cascone $\mathrm{P}$, Marini M: Fetal MRI as a complement to US in the evaluation of cleft lip and palate. Radiol Med 2011;116:11341148.

34 Kazan-Tannus JF, Levine D, McKenzie C, Lim KH, Cohen B, Farrar N, Busse RF, Mulliken JB: Real-time magnetic resonance imaging aids prenatal diagnosis of isolated cleft palate. J Ultrasound Med 2005;24:1533-1540. 